\title{
GL(1) Charged States in Twistor String Theory
}

\author{
Dimitri Polyakov ${ }^{\dagger}$ \\ Center for Advanced Mathematical Studies \\ and Department of Physics \\ American University of Beirut \\ Beirut, Lebanon
}

\begin{abstract}
We discuss the appearance of the GL(1) charged physical operators in the twistor string theory. These operators are shown to be BRST-invariant and non-trivial and some of their correlators and conformal $\beta$-functions are computed. Remarkably, the nonconservation of the $G L(1)$ charge in interactions involving these operators, is related to the anomalous term in the Kac-Moody current algebra. While these operators play no role in the maximum helicity violating (MHV) amplitudes, they are shown to contribute nontrivially to the non-MHV correlators in the presence of the worldsheet instantons. We argue that these operators describe the non-perturbative dynamics of solitons in conformal supergravity. The exact form of such solitonic solutions is yet to be determined.
\end{abstract}

$\dagger$ Presented at International Europhysics Conference i High Energy Physics, July 21st-27th 2005 Lisboa, Portugal 


\section{Introduction}

The hypothesis of the gauge-string correspondence, attempting to relate the gaugetheoretic and string degrees of freedom, is a long-standing problem of great importance. Remarkably, such a correspondence can be shown to occur both in the strongly coupled and the perturbative limits of Yang-Mills theory. Firstly, it is well-known that the AdS/CFT correspondence (e.g. see [1], [2], [3]) implies the isomorphism between vertex operators of string theory in the anti de Sitter backgrounds and the local gauge-invariant observables on the Yang-Mills side. On the other hand, recently it has been realized that some of the perturbative Yang-Mills scattering amplitudes are reproduced by correlators of string theory with the twistorial target space and the related topological B-model with Calabi-Yau target space [4], [5] The open string amplitudes of the twistor-string theory, supported on holomorphic curves in the projective space, have been shown to reproduce the perturbative gauge-theoretic amplitudes and the perturbative expansion on the Yang-Mills side have been shown to correspond to the D-instanton (or the worldsheet instanton) expansion in string theory [5], [6]. Typically, the worldsheet correlators of twistorial string theory involve the GL(1)-neutral vertex operators from both open and closed sectors. As already has been noted, some of these amplitudes reproduce the Yang-Mills perturbation theory, while others describe the conformal supergravity in the low-energy limit [7], [8] In this talk I show that, apart from the usual GL(1)-neutral operators, the twistor string theory also contains a set of physical GL(1)-charged vertex operators, argued to be related to the non-perturbative dynamics of the solitons appearing in conformal supergravity. These operators can be thought of as the second-quantized creation operators for the solitonic solutions of the conformal supergravity.

While these new vertex operators play no role in the MHV amplitudes, their role becomes important in the presence of the instantons (or, equivalently, in the non-MHV cases) and they do contribute nontrivially to both open and closed string amplitudes of the twistorial string theory. In this talk, we demonstrate the construction of such GL(1)charged BRST-invariant operators and derive the conditions for their BRST-invariance and non-triviality. At the first glance, appearance of such new physical vertices in twistorstring theory presents a problem as these states would seem to be in contradiction with the GL(1) gauge symmetry of the theory. One should remember, however, that in string theory BRST-invariance of some physical vertex operator does not automatically imply its gauge invariance. The simplest example is the local worldsheet supersymmetry in the NSR superstring model; e.g. the unintegrated picture -1 vertex operator of a photon is 
of course BRST-invariant; but the straightforvard application of the worldsheet supersymmetry generator to this vertex shows its non-invariance under the local supersymmetry transformations. Despite such a non-invariance, however, the relevant scattering amplitudes are invariant under the local supersymmetry, provided that one fixes the gauge parameter to vanish at the insertion points of the vertices. Such constraints on the gauge transformation parameter effectively reduce the gauge group, leading to appearance of the picture-changing operators in the amplitudes. The situation is somewhat similar in the twistor string theory, even though the complete and well-defined picture-changing formalism is yet to be developed for twistorial strings.

\section{$G L(1)$-charged operators and their amplitudes}

The worldsheet action for the twistor string theory in conformal gauge is given by

$$
\begin{array}{r}
S=\int d^{2} z\left(Y_{I} \nabla_{\bar{z}} Z^{I}+\bar{Y}_{I} \nabla_{z} \bar{Z}^{I}\right)+S_{c} \\
\nabla_{z}=\partial-A_{z} ; \nabla_{\bar{z}}=\bar{\partial}-A_{\bar{z}}
\end{array}
$$

where the supertwistors $Z^{I}=\left(\lambda^{\alpha}, \mu^{\dot{\alpha}}, \psi^{A}\right)$ are the homogeneous coordinates in the $C P^{3 \mid 4}$ target space, $Y_{I}=\left(\bar{\mu}_{\alpha}, \bar{\lambda}_{\dot{\alpha}}, \bar{\psi}_{A}\right)$ are conjugate to $Z, A_{a}(a=1,2)$ is the $G L(1)$ connection and $S_{c}$ is the action for a system of currents with $c=28$, necessary to cancel the conformal anomaly. In the open string case, $C P^{3 \mid 4}$ is reduced to $R P^{3 \mid 4}$ and $Z^{I}=$ $\bar{Z}^{I}, Y^{I}=\bar{Y}^{I}$ on the worldsheet boundary. When worldsheet instantons are absent, the stress-energy tensor and the $G L(1)$-generator are given by

$$
\begin{array}{r}
T=Y_{I} \partial Z^{I}+T_{c} \\
J_{G L(1)}=Y_{I} Z^{I}
\end{array}
$$

Using the OPEs:

$$
\begin{array}{r}
\lambda_{\alpha}(z) \bar{\mu}_{\beta}(w)=-\bar{\mu}_{\alpha}(z) \lambda_{\beta}(w) \\
\bar{\lambda}_{\dot{\alpha}}(z) \mu_{\dot{\beta}}(w)=-\mu_{\dot{\alpha}}(z) \bar{\lambda}_{\dot{\beta}}(w) \sim \frac{-\delta_{\alpha \beta}}{z-w}+\ldots \\
\psi_{A}(z) \psi_{B}(w) \sim \frac{\delta_{A B}}{z-w}+\ldots \\
\bar{\psi}_{A}(z) \bar{\psi}_{B}(w) \sim \frac{\delta_{A B}}{z-w}+\ldots
\end{array}
$$

one easily finds that, in the absence of the worldsheet instantons, the conformal dimensions and the $G L(1)$ charges of $Y$ and $Z$ are respectively

$$
\begin{array}{r}
h\left(Z^{I}\right)=0, h\left(Y_{I}\right)=1 \\
q\left(Z^{I}\right)=1, q\left(Y_{I}\right)=-1
\end{array}
$$


In addition, as it is clear from the form of the action (1), the $Y$ and $Z$ fields also can be attributed unit negative and positive ghost numbers respectively:

$$
g\left(Z^{I}\right)=-g\left(Y_{I}\right)=1
$$

with the ghost number current having the same form as as the $G L(1)$ generator (2), so essentially $g=q$. Given the stress tensor (2), there is no ghost anomaly, however the current does become anomalous when the worldsheet instantons are present. The $G L(1)$ neutral physical vertex operators of the theory in the unintegrated $b-c$-picture are given by:

$$
\begin{array}{r}
V_{\phi}=c j^{k} \phi_{k}(Z) \\
V_{f}=c Y_{I} f^{I}(Z), V_{g}=c \partial Z^{I} g_{I}(Z)
\end{array}
$$

with the additional constraints on the space-time fields: $\partial_{I} f^{I}=0, g_{I} Z^{I}=0$ and $j^{k}(z)$ being the dimension 1 currents from the current algebra, satisfying the usual OPE (suppressing the conventional factor of $i$ before the structure constants):

$$
j_{i}(z) j_{k}(w) \sim \frac{k \delta_{i k}}{(z-w)^{2}}+\frac{f_{i k l} j^{l}(w)}{z-w}+\ldots
$$

Here $V_{\phi}$ corresponds to the super Yang-Mills states while $V_{f}$ and $V_{g}$ describe the conformal supergravity sector. As the operators are $G L(1)$-neutral, the $G L(1)$ charges of $\phi_{k}, f^{I}, g_{I}$ are 0,1 and -1 respectively. When the worldsheet instanton number is zero $\left(\int d^{2} z \epsilon^{a b} F_{a b}=0\right)$, these are the only massless operators, contributing to perturbative correlation functions of the twistor string theory. In the presence of the worldsheet instantons, however, things change significantly. For the instanton number $d \neq 0$ the expression for the stress tensor (2) is deformed according to

$$
T \rightarrow T+\frac{d}{2} \partial J=T_{c}+Y_{I} \partial Z^{I}+\frac{d}{2} \partial Y_{I} Z^{I}
$$

implying the $G L(1)$ charge anomaly of the vacuum equal to $-d$. To compensate for it, the total $G L(1)$-charge of the vertices entering the correlator must be equal to $d$. Furthermore, the conformal dimensions of the fields also get twisted as

$$
\begin{gathered}
h\left(Z^{I}\right) \rightarrow h\left(Z^{I}\right)-\frac{d}{2}=-\frac{d}{2} \\
h\left(Y_{I}\right) \rightarrow h\left(Y_{I}\right)+\frac{d}{2}=1+\frac{d}{2}
\end{gathered}
$$


In general, the dimension of any space-time field $H_{q}(Z)$ carrying the $G L(1)$-charge $q$ is twisted according to

$$
h\left(H_{q}\right) \rightarrow h\left(H_{q}\right)-\frac{q d}{2}
$$

Such a twist of course does not change the dimensions of the $G L(1)$-neutral operators (6), but if we are able to find the charged $G L(1)$-operators commuting with the BRSToperator, the anomalous dimension changes must be taken into account. Firstly, consider the simplest case $d=2$ and the operator

$$
V_{2}=c v \partial Z^{I}: j_{i} j_{k}: H_{I}^{i k}(Z)
$$

with the space-time field $H_{I}^{i k}$ carrying the $+1 G L(1)$-charge. The total charge of the operator is +2 . Note that, unlike the open neutral operators (6), the operator (11) carries both the $R P^{3 \mid 4}$ and the current algebra indices. Using the relations (9), (10) it is easy to see that the operator (11) has conformal dimension zero. Let us check its BRST-invariance.The BRST operator is given by [8]

$$
Q=\int \frac{d z}{2 i \pi}\left(c T-b c \partial c+v Y_{I} Z^{I}+c u \partial v\right)
$$

In the $d=2$ instanton background the operator (11) has conformal dimension zero and it is a primary field provided that

$$
\begin{array}{r}
\operatorname{Tr}(H) \equiv H_{I}^{i k}(Z) \delta_{i k}=0 \\
f_{i k l} H_{I}^{k l}(Z)=0
\end{array}
$$

Therefore $V_{2}$ commutes with the $c T-b c \partial c$ part of $Q$. Commutation with the $\oint c u \partial v$ term of $Q_{b r s t}$ follows from two terms follows from the OPEs of the fermions $c(z) c(w) \sim(z-w)$ : $\partial c c:(w)$ cancelling the simple pole from the OPE of $u$ and $v$, so the full OPE of $c u \partial v$ and $V_{2}$ is non-singular. Finally,using $v(z) v(w) \sim(z-w): \partial v v(w)$, the commutation with the $\oint v J_{G L(1)}$ term gives

$$
\left\{\oint \frac{d z}{2 i \pi}: v Y_{I} Z^{I}:(z), V_{2}(w)\right\}=c v \partial v Z^{I} H_{I}^{i k}(Z(w)): j_{i} j_{k}:(w)=0
$$

provided that we impose the constraint on $H$ :

$$
Z^{I} H_{I}^{i k}(z)=0
$$


Now let us consider the question of the BRST non-triviality of $V_{2}$. The potential BRSTtriviality threat comes from the operator $W_{2}=c \partial Z^{I} j_{i} j_{k} H_{I}^{i k}(Z)$. Using the BRSTinvariance condition (15), one easily computes

$$
\left\{Q_{b r s t}, W_{2}\right\}=V_{2}+c v \partial Z^{I}: j_{i} j_{j}: Z^{J} \partial_{J} H_{I}^{i j}(Z)
$$

which implies that the $V_{2}$ operator is BRST notrivial, provided that

$$
Z^{J} \partial_{J} H_{I}^{i k}(Z) \neq 0
$$

. Equation (17) implies an important subtlety. Namely, at the first glance, it is not evident that, even if (17) is satisfied, this guarantees the non-triviality of the $V_{2}$-operator. Indeed, since the $H$-field (17) has a $G L(1)$ charge +1 and, generically, $Z^{J} \partial_{j}$ plays the role of the $G L(1)$-generator, naively one can write

$$
Z^{J} \partial_{J} H_{J}^{i k}(Z)=H_{J}^{i k}(Z)
$$

and then (16),(17) would seem to imply $2 V_{2}=\left\{Q_{b r s t}, W_{2}\right\}$. More generally, for any $H$-field of the $G L(1)$ charge $Q$ one would have

$$
Z^{J} \partial_{J} H(Z)=q H(z)
$$

In fact, however, the relations (18), and (19) are not always true - and whenever they are not fulfilled, $V_{2}$ is non-trivial. Let us address this question in more details. Whether (18) and (19) are true, depends completely on the form the $H$-fields depend on the homogeneous $Z$-coordinates. For instance, (18) and (19) are always true if $H$ is some homogeneous polynomial of $Z^{I}$, e.g. $H^{I_{1} \ldots I_{q}}=Z^{I_{1}} \ldots Z^{I_{q}}$, as it is easy to check. On the other hand, (18) and (19) are not satisfied if $\mathrm{H}$ contains any logarithmic dependence, e.g.

$$
\begin{array}{r}
H^{I_{1} \ldots I_{q}}(Z)=Z^{I_{1}} \ldots Z^{I_{q}} \log \left(\prod_{j=1}^{n} Z^{I_{j}}\right) \\
Z^{J} \partial_{J} H^{I_{1} \ldots I_{q}}(Z)=q H^{I_{1} \ldots I_{q}}(Z)+n Z^{I_{1}} \ldots Z^{I_{q}} \neq H^{I_{1} \ldots I_{q}}
\end{array}
$$

At this point, the following remark should be made. Naively, introducing of the logarithms in $H$ would not seem to be a good idea, since under the GL(1) transformation $Z \rightarrow t Z$ the logarithm is translated by $t$, seeming to imply that it doesn't have an associated well-defined $G L(1)$-charge and therefore we cannot talk definitely about $G L(1)$ charge 
and hence the definite conformal dimension of the H-field in (20). However we will show that, in the sense explained below, the logarithm of $Z$ can in fact be regarded as a field of the $G L(1)$ charge zero and the conformal dimension zero in any worldsheet instanton background. Namely, consider the instanton background $d=2 N$ and the associated stressenergy tensor for the $Y Z$-system:

$$
T(z)=Y_{I} \partial Z^{I}+N \partial\left(Y_{I} Z^{I}\right)=(N+1) Y_{I} \partial Z^{I}+N Z^{I} \partial Y^{I}
$$

Then the OPE of this tensor with $\log Z^{J}$ gives

$$
\begin{aligned}
& T(z) \log Z^{J}(w)=(N+1) \frac{\partial Z^{J}(w)}{Z^{J}}+N Z^{J}(w) \partial_{w} \frac{1}{Z^{J}(w)} \\
= & (N+1) \partial\left(\log Z^{J}(w)\right)-N \partial\left(\log Z^{J}(w)\right)=\partial\left(\log Z^{J}(w)\right)
\end{aligned}
$$

implying that $\log Z^{J}$ is a primary field of dimension 0 in any $d=2 N$ worldsheet instanton background. But then the equation (10), defining the correspondence between the $G L(1)$ charges and the anomalous conformal dimensions in the instanton backgrounds, implies that the $G L(1)$ charge of the $\log Z^{J}$ field must also be set to zero. This means that inclusion of the logarithms in the space-time $H$-fields $(17),(20)$ does not change their $G L(1)$ charges or conformal dimensions and at the same time protects their BRST non-triviality according to (17). Thus the coordinate dependence of the target space fields of the physical $G L(1)$ charged states always needs to include the logarithms.

Thus conditions $(13),(15)$, and (17) on the $H$-field ensure that the $V_{2}$-operator is physical, i.e. BRST-invariant and non-trivial, provided that the $H$ field contains the logarithmic dependence on the target space coordinates.

It is important that the explicit dependence of the $V_{2}$-operator on the $G L(1)$ ghost $v$ field protects it from having a simple pole due to the OPE with $J=Y_{I} Z^{I}$ because of its nonzero $G L(1)$ charge (provided that the constraint (15) is satisfied. The ghost number anomaly of the $u-v$ system is equal to -1 , as the $u$ and $v$ ghosts have dimensions 1 and 0 and therefore can be bosonized as $u=e^{-\chi}, v=e^{\chi}$ with $\chi$ being a free field with stressenergy tensor $T_{\chi}=\frac{1}{2}\left((\partial \chi)^{2}+\partial^{2} \chi\right)$. As the $Y-Z$ ghost anomaly for tree level correlator is equal to -2 for $d=2$, and the $G L$-neutral operators do not depend on $u$ and $v$, it appears that, unless some consistent picture-changing formalism is introduced, the only non-vanishing correlators (with the ghost anomaly cancelled) would involve not more than one $V_{2}$-operator with arbitrary number of $G L(1)$-invariant vertices. The form of dimension 0 BRST-invariant picture-changing operator lowering the $u-v$ ghost number by one unit 
can be found relatively easily from the integration over the moduli of the $A_{z, \bar{z}} G L(1)$ connection, present in the worldsheet action (1). The integration over the moduli of the worldsheet $G L(1)$ gauge field leads, as usual (along with the standard condition that the basis of the moduli is orthogonal to the gauge slice) to the insertion of the picture-changing operators inside the correlation functions, with the form

$$
\Gamma(z)=u \delta\left(J_{G L(1)}\right)=u \delta\left(Y_{I} Z^{I}\right)
$$

Here the $u$ ghost has the +1 dimension and the delta-function of the $G L(1)$-current is of the dimension -1 , so the total dimension of $\Gamma$ is zero. The BRST-invariance of $\Gamma$ can be easily checked by using

$$
\begin{array}{r}
\left\{Q_{b r s t}, u\right\}=Y_{I} Z^{I} \\
{\left[Q_{b r s t}, Y_{I} Z^{I}\right]=0} \\
: Y_{I} Z^{I} \delta\left(Y_{I} Z^{I}\right): \sim 0
\end{array}
$$

Note that the $R$-operator, introduced in the paper by Berkovits and Motl [8] and which increases the $u-v$ ghost number by 1: $R=v \delta(r(z)-1)+c \partial r \delta(r(z)-1)$ where $r$ is the scale factor for the $Z$-field, should in fact be regarded as the inverse rather than the direct picture-changing operator. Note that, unlike the case of $\Gamma$, it is not yet clear how the $R$-operator can be derived from the first principles, i.e. from the the integration over moduli. Using the appropriate insertions of $\Gamma$ and $R$-operators in the correlators, one can in principle consider the amplitudes with arbitrary number of the $V_{2}$-vertices.

As an example, consider the 3 -point correlation function of the $V_{2}$-operator with 2 gluon vertices at $d=2$. Simple computation gives

$$
\begin{array}{r}
<c v \partial Z^{I}: j_{i} j_{k}:\left(z_{1}\right) H_{i j}^{I}(Z) c j^{l}\left(z_{2}\right) \phi_{l}(Z) c j^{m} \phi_{m}\left(z_{3}\right)> \\
=\int d^{4} x d^{8} \theta \int_{D_{x, \theta}} d Z^{I} H_{I}^{i k}(Z) \phi_{i} \phi_{k}
\end{array}
$$

where the degree 1 curve $D_{x, \theta}$ is defined through $\mu^{\dot{\alpha}}=x^{\alpha \dot{\alpha}} \lambda_{\alpha}, \psi^{A}=\theta^{A \alpha} \lambda_{\alpha}$ with $\lambda$ being the homogeneous coordinates and $(x, \theta)$ the moduli of the curve. Let us now turn to the general case of $d=2 N$. Repeating the above arguments, it is easy to see that the spectrum of massless physical GL(1)-charged states is given by the vertex operators of the form:

$$
\begin{array}{r}
V_{2 N}(w)=c v \partial Z^{I_{1}} \ldots \partial Z^{I_{P}}\left[H_{I_{1} \ldots I_{P}}^{i_{1} \ldots i_{m(P)}}(Z(w))\right]_{q_{G L(1)}(H)=2 N-P}: j_{i_{1} \ldots . j j_{i_{m(N, P)}}}:(w) \\
m(N, P)=2 N^{2}-P+1 ; P \leq 2 N
\end{array}
$$


The $I_{k}$ are the target space indices of $R P^{3 \mid 4}$ while the $i_{k}$ indices are related to the current algebra (7). Each of these operators has the total $G L(1)$ charge $q=2 N$ so that one insertion of $V_{2 N}$ in correlation functions compensates for the $Y-Z$ ghost anomaly. The space-time $H$-fields are allowed to have $G L(1)$ charges equal to $q_{G L(1)}=2 N-P, P=$ $0, \ldots 2 N$. As it is easy to check, the number $m(N, P)$ of the dimension 1 current insertions ensures that the total conformal dimensions of the operators (25) are all equal to zero. They are symmetric in both the target space and the current algebra indices. The BRST invariance and non-triviality conditions are derived completely similarly to the case of $V_{2}$. The BRST-invariance of $V_{2 N}$ requires

$$
\begin{aligned}
Z^{I_{1}} H_{I_{1} \ldots I_{P}}^{\left.i_{1} \ldots i_{m}(N) P\right)}(Z) & =0 \\
H_{I_{1} \ldots I_{P}}^{i_{1} \ldots i_{k} \ldots i_{l} \ldots i_{m(N, P)}}(Z) \delta_{i_{k} i_{l}} & =0 \\
f_{i i_{k} i_{l}} H_{I_{1} \ldots I_{P}}^{i_{1} \ldots i_{k} \ldots i_{l} \ldots i_{m(N, P)}}(Z) & =0
\end{aligned}
$$

(as $H$ is symmetric in the current indices, the second and the third identities hold for any pair of $i_{k}$ and $i_{l}$ ). The BRST non-triviality of $V_{2 N}$ requires

$$
Z^{J} \partial_{J} H_{I_{1} \ldots I_{P}}^{i_{1} \ldots i_{m(N) P)}}(Z) \neq 0
$$

As previously, the last equation also must be supplemented with the condition that the $Z$-dependence of the $H$-fields must contain logarithms.

\section{Conclusion and Discussion}

We analyzed the properties of the $G L(1)$-charged vertex operators in twistor string theory, emerging in the presence of the worldsheet instantons. But the main question remains - what physical degrees of freedom do these operators correspond to? Once we know that the perturbative massless spectrum of the twistor string theory is limited to the neutral $G L(1)$ states (corresponding to $N=4$ super Yang-Mills theory and the conformal supergravity), then what is the role played by the tower of the charged massless states? Below we shall attempt to present some arguments that these states are related to the non-perturbative sector of the twistor-string theory, possibly corresponding to the collective coordinates of non-perturbative solitonic (or brane-like) solutions of the conformal supergravity theory. To justify such a conjecture, let us make an excursion to the NSR superstring theory, in order to point out some important analogies with twistorial strings. While the conventional perturbative vertices in twistor string theory are $G L(1)$ neutral (which usually is the consequence of their BRST properties) the BRST-invariance 
of the charged vertices is ensured by their explicit dependence on the $v$ ghost field,i.e. these operators are essentially mixed with $G L(1)$-ghosts. Moreover, the $G L(1)$-charge is not conserved in the interactions involving the $V_{2 N}$-vertices, as has been demonstrated by the analysis of their renormalization group flows (34). At the same time, the operator algebra involving the products of $V_{2 N}$-operators with $G L(1)$-neutral states still has a remarkably simple structure (30). In NSR superstring theory there exists a set of physical operators with remarkably similar properties. These operators are also non-invariant under local worldsheet supersymmetry, however their coupling with superconformal ghosts (which cannot be removed by picture-changing transformations and therefore is not an artefact of a picture choice) ensures their physical BRST properties. These operators exist in both open and closed string sectors and are related to non-perturbative dynamics of branes. Examples of such open-string operators are given by the five-form state at pictures -3 and +1 :

$$
\begin{array}{r}
V_{5}^{(-3) \text { open }}=H^{m_{1} \ldots m^{5}}(k) \oint \frac{d z}{2 i \pi} e^{-3 \phi} \psi_{m_{1}} \ldots \psi_{m_{5}} e^{i k X} \\
V_{5}^{(+1) \text { closed }}=H^{m_{1} \ldots m^{5}}(k) \oint \frac{d z}{2 i \pi} e^{\phi} \psi_{m_{1} \ldots \psi_{m_{5}} e^{i k X}+\text { ghosts }} \\
k^{\left[m_{1}\right.} H^{\left.m_{2} \ldots m_{6}\right]} \neq 0
\end{array}
$$

This five-form state exists at ghost pictures above +1 or below -3 but, say, not at the picture zero, so its mixing with superconformal ghosts is essential. The zero-momentum part of the picture -3 appears as a five-form extension in the picture-twisted space-time superalgebra of NSR superstring theory:

$$
\begin{array}{r}
\left\{T_{\alpha}, T_{\beta}\right\}=\gamma_{\alpha \beta}^{m} P_{m}+\gamma_{\alpha \beta}^{m n} Z_{m n}+\gamma_{\alpha \beta}^{m_{1} \ldots m_{5}} Z_{m_{1} \ldots m_{5}} \\
T_{\alpha}=\oint \frac{d z}{2 i \pi}\left(e^{\frac{-3}{2} \phi}+e^{\frac{-\phi}{2}}\right) \Sigma_{\alpha} \\
P_{m}=\oint \frac{d z}{2 i \pi} e^{-\phi} \psi_{m} ; Z_{m n}=\oint \frac{d z}{2 i \pi} e^{-2 \phi} \psi_{m} \psi_{n} \\
Z_{m_{1} \ldots m_{5}}=\oint \frac{d z}{2 i \pi} e^{-3 \phi} \psi_{m_{1}} \ldots \psi_{m_{5}}
\end{array}
$$

where $\Sigma$ is the spin operator for matter fields, $\phi$ is the bosonized superconformal ghost and $\psi$ are the NSR worldsheet fermions. The superalgebra (36) is isomorphic to the dimensionally reduced M-theory algebra, or the SUSY algebra of the $d=11$ supergravity [9]. It has been pointed out [9] that the central $p$-form terms in the SUSY algebra always correspond to the presence of extended objects (p-branes) in the theory. Thus one shall 
attempt to relate the 2 -form and the 5 -form vertices (36) to topological charges of the Mbranes, i.e. purely perturbative physical vertices account for the dynamics and topology of essentially non-perturbative objects (e.g. the non-perturbative solutions of $d=11$ supergravity). Moreover, many analogues of these states exist in the closed string sector, some of them can be obtained e.g. by taking the product of the left 5 -form and the photon-type right-moving part:

$$
\begin{array}{r}
V_{5}^{\text {closed }}=H^{m_{1} \ldots m_{6}} \int d^{2} z\left(e^{\phi-\bar{\phi}}+e^{-3 \phi-\bar{\phi}}\right) \psi_{m_{1}} . . \psi_{m_{5}} \bar{\psi}_{m_{6}} e^{i k X}+\text { ghosts } \\
k_{m_{6}} H^{m_{1} \ldots m_{5} m_{6}}=0 \\
k^{\left[m_{7}\right.} H^{\left.m_{1} \ldots m_{5}\right] m_{6}} \neq 0
\end{array}
$$

Here the 6-tensor $H$ is antisymmetric in the first 5 indices. Using the BRST conditions (31) on $H$, one can eliminate half of its independent components. Then for each fixed orientation $m_{1} \ldots m_{6}$ of $\mathrm{H}$ in the space-time the constraints (37) imply that there are six independent degrees of freedom $\lambda^{t}, t=1 \ldots 6$ per orientation and the momentum $k$ must me orthogonal to the $m_{1} \ldots m_{6}$ directions (the exact relation between $\lambda$ and $H$ is given in [10]) Using the above conditions one can calculate the effective action for the fixed orientation of $H$ to be given by

$$
\begin{array}{r}
S_{e f f} \sim \int d^{4} x e^{-\varphi} \sqrt{\operatorname{det}\left(\eta_{a b}+\partial_{a} \lambda^{t} \partial_{b} \lambda^{t}\right)} \\
a, b=0 . .3
\end{array}
$$

i.e. the DBI effective action for D3-brane. On the other hand, the open string $V_{5}$ operators are responsible for the RR-coupling terms and topological charges of various brane configurations. Finally, the OPE algebra of the $V_{5}$ operators (both in the open and the closed string cases) with usual perturbative NSR vertices has a structure totally similar to (30) of the twistor string case, i.e. [10]

$$
V_{5}(z) V(w) \sim \frac{1}{z-w} C V_{5}\left(\frac{z+w}{2}\right)
$$

where $\mathrm{C}$ are the structure constants and $\mathrm{V}$ is either a photon, a dilaton, a graviton or an axion. We have brought up the recollection of the NSR scenario to emphasize our claim that the $V_{2 N}$-vertices discussed in this letter have properties very similar to (35), (36) and (39) correspond to the non-perturbative solitonic objects that should appear in the conformal supergravity. At present, such solitonic (or brane-type) solutions have not yet been discussed in the context of conformal supergravity and it would be an interesting problem 
to explore. We hope to elaborate on it in the future works. The existence of physical $V_{2 N}$-operators seems to clearly predict the possibility of such non-perturbative solutions in the worldsheet instanton backgrounds. Once the conformal supergravity solitons are found, it would be important to calculate the effective actions governing their dynamics, which should be somewhat of the DBI-type. Unfortunately, at present we do not yet have such a formalism, and its construction would clearly need some further efforts. 


\section{References}

[1] S. Gubser, I. Klebanov, A.M. Polyakov, Phys. Lett. B428, 105 (1998)

[2] J. Maldacena, Adv. Theor. Math. Phys. 2, 231 (1998)

[3] E. Witten, Adv. Theor. Math. Phys, 2, 253 (1998)

[4] E. Witten, hep-th/9112056

[5] E. Witten,hep-th/0312171, Commun. Math. Phys.252:189(2004)

[6] F. Cachazo, P. Svrcek, E. Witten, hep-th/0406177, JHEP 0410:074 (2004)

[7] N. Berkovits, E. Witten, hep-th/0406051, JHEP 0408:009(2004)

[8] N. Berkovits, L. Motl, hep-th/0403187, JHEP 0404:056 (2004)

[9] J.Azcarraga, J.Gauntlett, J.Izquierdo, P.Townsend, Ph.Rev.Lett. D63(1989)2443

[10] D. Polyakov, hep-th/0406079, to appear in IJMPA 